\title{
Spider toxin SNX-482 gating modifier spontaneously partitions in the membrane guided by electrostatic interactions
}

\author{
Guido Mellado ${ }^{1,2}$, Jose Antonio Garate ${ }^{1,3 *}$ and Alan Neely ${ }^{1, *}$ \\ ${ }^{1}$ Centro Interdisciplinario de Neurociencias de Valparaíso, Facultad de Ciencias, Universidad de Valparaíso, \\ Valparaíso, Chile. \\ 2Doctorado en Biofísica y Biología Computacional, Facultad de Ciencias, Universidad de Valparaíso, Valpa- \\ raíso, Chile. \\ ${ }^{3}$ Millennium Nucleus in NanoBioPhysics, Facultad de Ciencias, Universidad de Valparaíso, \\ Valparaíso 236 0102, Chile. \\ * Correspondence: jose.garate@uv.cl (JAG); alan.neely@uv.cl (AN)
}

\begin{abstract}
Spider toxin SNX-482 is a cysteine-rich peptide that interferes with calcium channel activity by binding to voltage-sensing domains of the Cav2.3 subtype. Two general binding mechanisms are present in nature: direct binding from the aqueous phase or through lateral diffusion from the membrane, the so-called reduction in dimensionality mechanism. In this work, via coarse-grained and atomistic molecular dynamics simulations, we have systematically studied the spontaneous partitioning of SNX-482 with membranes of different anionic compositions and explored via diffusional analysis both binding mechanisms. Our simulations revealed a conserved protein patch that inserts within the membrane, a preference for binding towards partially negatively charged membranes, and that electrostatics drives membrane binding. Finally, diffusivity calculations showed that the toxin diffusion along the membrane plane is an order of magnitude slower than the aqueous phase suggesting that the critical factor in determining the SNX-482-Cav2.3 binding mechanism is the affinity between the membrane and SNX-482.
\end{abstract}

Keywords: Calcium channel, gating modifier, SNX-482, cysteine-rich peptide. Membrane partitioning

\section{Introduction}

Among animal species that use poison to kill or paralyze prey, spiders emerge as the richest natural sources of toxic peptides. This group of peptides can be classified based on their number of disulfide bonds that range from one to seven [1]. The most abundant group of spider toxins share a structural pattern called inhibitor cystine knot (ICK) characterized by disulfide bridges. ICK structural motif is defined by an antiparallel betasheet stabilized by a ring formed by two disulfide bridges (Cys1-Cys4 and Cys2-Cys5) and a disulfide bond (Cys3-Cys6) that passes through in the ring [2,3]. These internal bridges confer exceptional resistance to high temperatures $\left(80 \mathrm{C}^{\mathrm{o}}\right)$, proteases degradation, extreme $\mathrm{pH}$, and organic solvents [4]. ICKs preferred targets are voltage-dependent ion channels $[5,6]$. Channels from the superfamily of voltage-gated channels are endowed with four voltage-sensing domains (VSD) surrounding a single pore domain (PD) at the center of symmetry. Each VSD consists of four transmembrane helices flanked by two intracellular segments. The C-terminal end of each VSD connects to two transmembrane segments contributing to the PD. ICK toxins modulate voltage-gated channel activity by two mechanisms: preventing ion flow by occluding the channel as is the case of Charybdotoxin inhibiting $\mathrm{Ca}+2$ activated $\mathrm{K}+$ channels called pore-blocker [7] or interfering with voltage-dependent gating such as Hanatoxin (HaTx) and VSTx1 [8,9] named Gating modifiers toxins (GMTx). In contrast to pore-blockers that diffuse through the aqueous phase, GMTx meander along the membrane plane to reach their target. Most spider GMTx had 
adopted the "reduction-of-dimensionality" (RD) strategy [10] that is preceded by an abrupt increase in local concentration mediated by non-specific binding to the outer leaflet of the membrane. The formation of the ternary complex with the channel protein is thought to be favored by reactants diffusion in two dimensions. However, there is no direct evidence supporting this view. Membrane partition may also increase the local concentration dramatically. The question then arises to what extent the apparent binding affinity measured experimentally is dominated by the significant free energy change $(\Delta \mathrm{G})$ of the toxin partitioning into the membrane [11,12]. How does the membrane lipid composition contribute to this process? Previous studies have established a preference for membranes rich in anionic phospholipids[13,14]. However, the requirement for anionic lipids for membrane partitioning remains controversial since GMTx with a high affinity to neutral or mixed membrane models has been identified. $[8,12,15]$. Since most studied GMTx are positively charged, it is tempting to conclude that the net charge is relevant to toxin-membrane interaction, but membrane-access mechanisms cannot be generalized to all GMTx, and they must be investigated case-by-case. An interesting example is SNX482 , which is unique in having a negative net charge (-2). SNX-482 is a 41 residues GMTx isolated from the African tarantula venom, Hysterocrates gigas [16] and is the only reported potent antagonist of the neuronal calcium channel subtype Cav2.3 [17]. SNX-482 inhibits activation and is thought to interact with VSD of repeats 3 and 4 of Cav2.3 channels. The binding of SNX-482 to the VSD would hinder its activation, shifting voltage dependence by about $+60 \mathrm{mV}$. VSD-Toxin interaction can be reversed by strong depolarization, an observation predicted by a model in which SNX-482 prefers the resting state of the VSD[18]. The enthusiasm for SNX-482 as a therapeutic agent was somewhat diminished by discovering that it also blocks potassium channels Kv4.2 and Kv4.3 [19]. However, recent research could position SNX-482 as an interesting immunomodulatory molecule for anticancer immunotherapy. [20].

This work extensively investigates the spontaneous partitioning of SNX-482 into lipid bilayers of different anionic compositions using coarse-grained (CG) and all-atom (AT) molecular dynamics simulations. As a whole, our simulations consistently revealed a conserved protein patch that inserts within the membrane. Furthermore, atomistic simulations exhibited a preference for partially negatively charged bilayers. Despite a net charge of -2 , energy calculation indicates that binding to the membrane is driven by electrostatic, probably because of the large number of polar residues being inserted. Finally, diffusivity calculations showed that the toxin diffusion along the membrane plane is at least an order of magnitude slower than in the aqueous phase, suggesting that reducing dimensionality does not aid SNX-482-Cav2.3 binding. Most likely, efficient membrane binding is a strategy to increase membrane concentration that reduces the apparent affinity for the receptor and thus, being an effective toxin while relaxing the structural requirement for tight binding to the receptor.

\section{Materials and Methods}

\subsection{SNX-482 modeling}

Template structures for SNX-482 were searched with BLAST [21], and highly similar sequences with known 3D structures were aligned (see Figure 1A). Jingzhaotoxin-XI (PDB code: $2 \mathrm{a} 2 \mathrm{v}$ ) was selected as a template, and a comparative model of SNX-482 was generated with MODELLERv9.2 [22]. Three single structures from the NMR ensemble were selected and equilibrated by all-atom molecular dynamics (MD) simulations for 40 ns, each. The most representative structure of Jingzhaotoxin-XI was obtained by a K-mean clustering (RMSD Ca-based with a $0.2 \mathrm{~nm}$ cutoff) analysis from the MD conformational ensemble employing the gromos++ software package[23] and then selected a template for the SNX-482 modeling. Comparative models ( 100) were generated by satisfying spatial restraints via MODELLERv9.2. These models were grouped into three central clusters, whose centroids were subjected to $40 \mathrm{~ns}$ MD simulations. The final SNX-482 structural model corresponds to the centroid of the most representative cluster of the MD 
trajectories, as summarized in Supplementary Figure S1. A depiction of the final model, emphasizing its distribution of positive charges, is shown in figure $1 \mathrm{~b}$.

\subsection{Coarse-grained Molecular Dynamics Simulations}

Coarse-Grained (CG) MD simulations were performed with the CUDA accelerated version of GROMACS 2021 [24], employing the Martini 3 force field [25]. All simulations were carried out in the NPT ensemble, a $1 \mathrm{~atm}$ and $298 \mathrm{~K}$ in semi-anisotropic conditions, with pressure control via the Parrinello-Rahman barostat [26] and temperature control with the velocity-rescale thermostat. A $1.1 \mathrm{~nm}$ cutoff was employed for real-space electrostatics and Van der Waals (VdW) interactions with a smoothing function applied within. Long-range Coulombic interactions were computed with the Particle-Mesh Ewald method (PME). A time-step of $20 \mathrm{fs}$ was utilized for the MD integrator. The SNX-482 was converted to CG particle with Martinize [27]. Toxin configuration was constrained by an elastic-network formulation with lower and upper cutoffs of $0.5 \mathrm{~nm}$ and $0.9 \mathrm{~nm}$, respectively. A force constant of $700 \mathrm{~kJ} /\left(\mathrm{mol} \mathrm{nm}^{2}\right)^{-1}$ was used.

Membrane bilayer parches were built via the CHARMM-GUI with the Martini Bi-

(a)

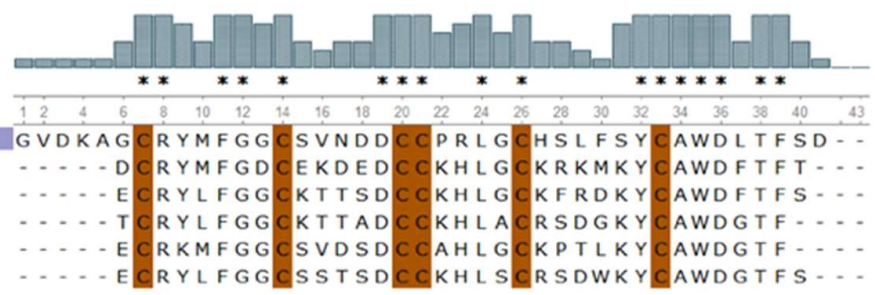

(b)

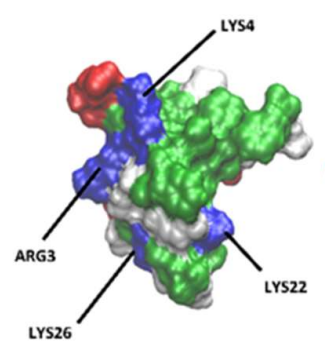

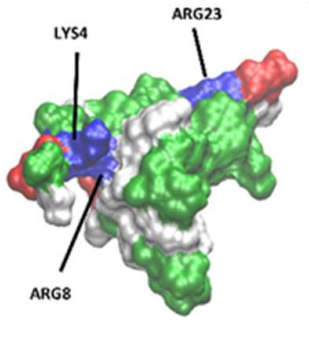

(c)
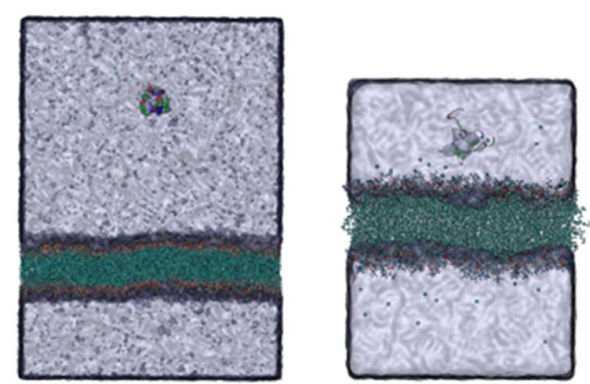

Figure 1. Comparative Modeling SNX-482. (a) Alignments of the five ICK toxins with the highest identity score to SNX-482. Identity score is included for each toxin. SNX-482(Omega-theraphotoxin-Hg1a) is highlighted in purple. Bars at the top of the alignment depict the percent identity at each position of the sequence. Conserved cysteines are highlighted in brown. (b) Surface representations of the Jingzhaotoxin-XI (left) and the SNX-482 (right) colored by residue type: positively charged (blue), negatively charged (red), and polar (white), hydrophobic (green). Both toxins' structures are in the same orientation according to structural alignment. (c) The representative initial simulation system of the CG model (left) and the all-atom setup (right).

layer Maker module [28]. Mixtures of neutral (1-palmitoyl-2-oleoyl-sn-glycero-3-phosphocholine or POPC) and charged (palmitoyl-2-oleoyl-sn-glycero-3-phospho-rac-(1-glycerol) or POPG) at five different ratios were generated 1) POPC only, 2) POPC:POPG :1:3, 3) POPC:POPG :1:1, 4) POPC:POPG :3:1 and 5) POPG only. In all cases, counter ions were added to neutralize the systems. On average, the CG systems consisted of 109531 supraparticles with an average box size of $21 \times 21 \times 29 \mathrm{~nm}$ (see Figure 1c left). For each membrane 
composition, four independent simulations with the toxin placed at $12 \mathrm{~nm}$ from the outer leaflet of the membrane and in random orientations were carried out for $1 \mu \mathrm{s}$

\subsection{All Atom Molecular Dynamics Simulations}

All-atom (AT) MD simulations were carried out with the NAMDv2.13 CUDA accelerated simulation engine[29], the CHARMM36 parameter set for proteins, ions, and lipids, and the TIP3P water model [30]. All simulations were carried out at the NPT (1 atm and 298K) ensemble with Langevin dynamics for temperature and semi-anisotropic piston fluctuation control. Long-range electrostatics were computed with the PME method with a relative tolerance of $1 \times 10^{-6}$ and a cutoff of $1.2 \mathrm{~nm}$ for Van der Wall (VdW). Real-space electrostatic interactions and a switching function were applied between 1.0 and $1.2 \mathrm{~nm}$. We used multiple time-step algorithms that consisted of $2 \mathrm{fs}$ steps for bonded interactions and real space non-bonded interactions and 4 fs steps for the reciprocal space electrostatic interactions. All bonds involving hydrogens were constrained via the SHAKE algorithm [31].

To investigate membrane partitioning of the toxin, we generated five bilayer patches of $\sim 11.5 \times 11.5 \mathrm{~nm}$ containing 308 lipids with an axial length of $8.4 \mathrm{~nm}$, employing the same compositions as for CG simulations. Counter ions were added to neutralize the systems. In these simulations, toxins were placed at $\sim 2.5 \mathrm{~nm}$ from the outer leaflet of the membrane with different initial poses. The z-axes in all the simulation systems were set normal to the membrane plane. Overall, more than $30 \mu$ s of simulation time were performed in this study. A depiction of representative systems for the CG (left), and atomistic (right) systems are presented in figure $1 \mathrm{C}$.

\subsection{Membrane partitioning and Insertion probability:}

Without previous knowledge of the docking pose of SNX-482 towards the membrane and its binding mode with different lipid compositions, we analyzed this process as follows. We monitored the distance along the z-axis between the toxin's center of mass $(\mathrm{COM})$ and the COM of the membrane (see figures $3 a, 4 a)$. Once this distance reached a plateau, we computed the fraction of time each residue was inserted within the membrane. To this end, the COM of each phospholipid head from both sides of the membrane was fitted to a straight line $(z=a x+b)$. An insertion event was counted when the $z$ coordinates of the COM of a residue fell below $z$, and the insertion probability of residues (Ip) is the number of insertion events it undergoes divided by the number of frames in the trajectory. For each MD simulation, we defined an Insertion Score (IS) as follows:

$$
\mathrm{IS}=\sum_{i=1}^{N R} \mathrm{Ip}
$$

where NR is the total number of residues of SNX-482, the first insertion event to be computed was the one that occurred when the distance of the toxin COM and $\mathrm{z}$ was less than $0.5 \mathrm{~nm}$. This requirement excludes spurious collisional contact not related to a membranebinding process.

\subsection{Diffusion coefficient calculation}

The diffusion coefficient in 3D (water) and 2D (membrane) were calculated from mean squared displacements (MSDs) using the Einstein relation:

$$
D=\frac{1}{2 d} \lim _{t \rightarrow \infty} \frac{\left\langle\left(r\left(t_{0}+t\right)-r\left(t_{0}\right)\right)^{2}\right\rangle_{0}}{2 t}
$$

$$
\left\langle\left(r\left(t_{0}+t\right)-r\left(t_{0}\right)\right)^{2}\right\rangle_{t_{0}}=M S D
$$

where d corresponds to the number of dimensions ( 3 and 2 for bulk and lateral diffusion, respectively); $r$ is the (unwrapped) position vector of the center of mass (COM) at time $t$. Averaging was performed over multiple windows and times origins ( $\left.\mathrm{t}_{0}\right)$. For bulk diffusion, a $1 \mu \mathrm{s}$ MD in pure water was performed. Recent reports have described that finitesize effects when employing periodic boundary conditions and asymmetric box shapes (typical conditions for membrane simulations) arise in lateral diffusion coefficients within 
membranes in MD simulations [32]. Therefore, for lateral diffusion calculations, the periodic box of the last configuration from the MD with the highest IS was enlarged to a lateral size of $16 \mathrm{~nm}$ and the simulation time was extended to $2 \mu \mathrm{s}$. From these diffusion constants $\left(D^{\text {sim }}\right)$, we extrapolated lateral diffusion constants to an infinite box size $\left(D^{\infty}\right)$ employing a Bayesian method (https://diffusion.lobos.nih.gov/bayes.html) that employs the Periodic Safmann-Delbrück model. We also computed lateral diffusion coefficients for the lipids (COM of polar heads). For more details on the specific parameters used for these calculations, please refer to supplementary tables S1 and S2.

\section{Results and Discussion}

The general aim of the present study was to determine if the negatively charged SNX482 partitions into lipids membranes and investigate the importance of the anionic charge composition in this process. Many GMTx share a conserved hydrophobic patch surrounded by a ring of positively charged residues that have been proposed to promote peptide affinity for negatively charged lipid membranes $[8,33,34]$. Note that all sequenced aligned but SNX-482 share five positively charged residues (figure 1a) and that the comparative model of SNX-482 shows only three positive charges within the vicinity of the hydrophobic patch (see figure 1b). This raises the question about the ability of SNX-482 to interact with the membrane despite having an incomplete ring of positive charges and how negatively charged lipid may influence the binding of a peptide carrying a net negative charge.

\subsection{SNX-482 partitions into partially anionic membranes in a conserved manner.}

To investigate in detail, membrane partition we carried out molecular dynamic simulation in CG and AT resolutions of SNX-482 in the presence of lipid bilayer with different ratios of neutral (POPC) and anionic lipids (POPG). We considered two parameters to evaluate membrane insertion: 1) the fraction of simulation in which at least one insertion event was observed and 2) the insertion score detailed in the method section. Table 1 summarizes the results of four simulations in each condition, i.e., lipid composition, and for each resolution level, i.e., CG or AT. For CG simulations, we did not observe insertion events in any of the four simulations carried out with neutral membrane (POPC). Insertion events were observed in all other conditions. More notable, when considering only simulation with insertion events, IS values fall within the error margins for all anionic membranes, from POPC: POPG (3:1) to full POPG. This observation would lead us to conclude that anionic lipids are necessary for the toxin insertion process. However, there was a successful membrane insertion event in one of the AT MD simulations with a neutral membrane. Thus, membrane binding, although disfavored, is still possible without anionic lipids.

Table 1. Insertion Score (IS) of SNX-482 in lipid bilayers of different POPC/POPG ratio.

\begin{tabular}{lrcrc}
\hline \multirow{2}{*}{ Lipid composition } & \multicolumn{2}{c}{ Coarse grain } & \multicolumn{2}{c}{ All-atom } \\
\cline { 2 - 5 } & \multicolumn{1}{c}{ IS } & Bindings/Run & \multicolumn{1}{c}{ IS } & Bindings/Run \\
\hline POPC & 0 & $0 / 4$ & $2.7 \pm 2.5$ & $1 / 4$ \\
POPC:POPG (3:1) & $5.4 \pm 2.3$ & $4 / 4$ & $13.4 \pm 2.2$ & $4 / 4$ \\
POPC:POPG (1:1) & $7.9 \pm 1.1$ & $4 / 4$ & $3.9 \pm 2.3$ & $2 / 4$ \\
POPC:POPG (1:3) & $4.7 \pm 3.1$ & $2 / 4$ & $5.5 \pm 2.2$ & $2 / 4$ \\
POPG & $6.8 \pm 2.3$ & $3 / 4$ & $1.3 \pm 0.6$ & $1 / 4$ \\
\hline
\end{tabular}

IS: insertion score, Bindings/Run: the ratio of simulations with at least one insertion event occurring while the membrane-toxin distance was less than $0.5 \mathrm{~nm}$ 
We also observed a significant preference over partially charged membranes in AT simulations, particularly for the 3:1 condition, and that fully charged membrane also disfavors membrane insertion. Overall, IS appears more sensitive to the membrane composition in AT simulation than in CG. A partial explanation may be that COM values for each residue are closer to the protein backbone in CG, and thus, a fraction of shallower insertion event counted in AT is missing in CG. In AT simulations, IS appears very sensitive to the ratio of neutral to anionic lipids, reaching an optimum with a 3:1 ratio, about three-fold higher than the other mixed membranes. In contrast, Pure POPC or POPG seems equally unfavorable to membrane partition. A preference for a partially charged membrane over neutral ones has been observed experimentally with Protx II using plasmon resonance and all-atom simulation [14].

To explore in more detail the differences between the CG and AT descriptions, we computed the average Ip for each residue for all simulations in which there was a successful insertion event, regardless of the membrane composition (Figure 2A). Overlaying color-coded Ip values over a surface representation of SNX-482 allows us to visualize the global binding pose (figure $2 \mathrm{~b}$ ). These results show that membrane partitioning residues are conserved regardless of membrane type or resolution level. In both cases, the highest Ip values are distributed in the same two regions of the amino acid sequence. The first segment span from residues 9 to 14 , and the second encompasses from residue 20 to 35 .

(a)
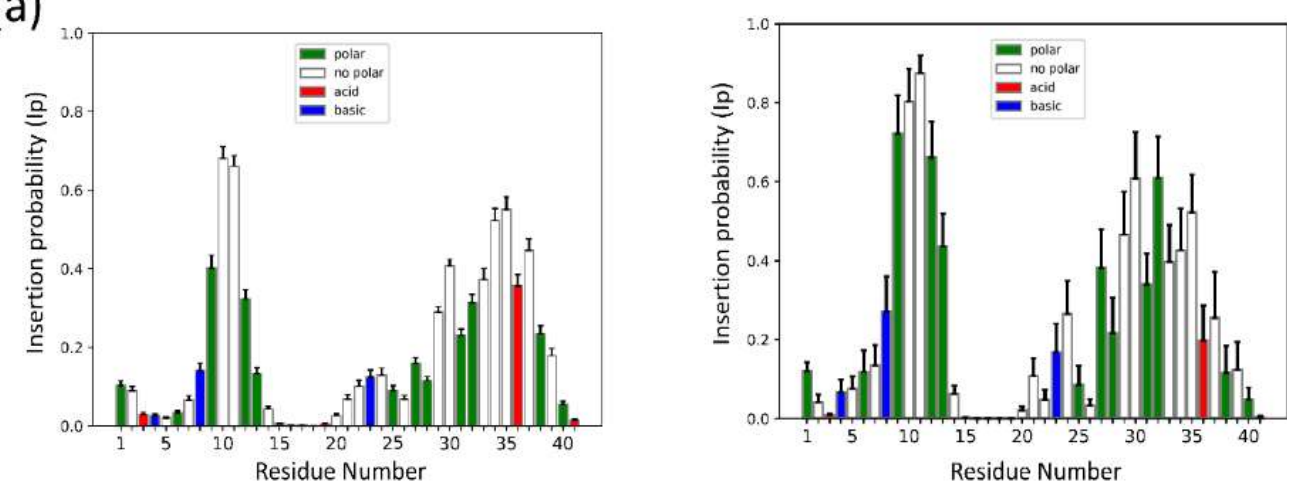

(b)
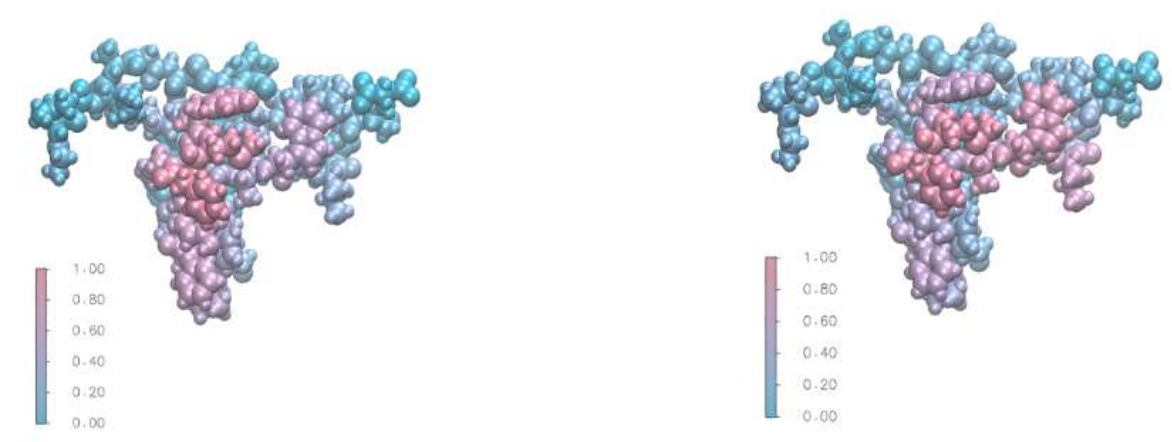

Figure 2. The global insertion probability (Ip) for coarse grain (CG) and all atom (AT) MD simulations. (a) The CG (right) and AT (left) insertion probability of all MD simulations where there was successful insertion $n=13$ (CG) and $n=10$ (AT). (b) VdW representations of SNX-482 coloring by probably of all MD simulations where there was successful insertion. AT (right) and CG (left). 
The insertion process was investigated in more detail on simulations with the membrane composition that reported the largest proportion of insertion events. These correspond to POPC: POPG ratios of 1:1 and 3:1 for CG and AT, respectively. The time evolution, average Ip for each residue, and binding pose are shown for these two cases: Figure 3 corresponds to CG simulation and Figure 4 to AT MD. Results for the other membrane compositions are reported in supplementary figures (S2 to S12). In both systems, the toxin comes into close contact with the membrane and reaches a plateau (below $0.5 \mathrm{~nm}$ ) that remains stable for the final $200 \mathrm{~ns}$ of the simulation (figures .3a and $4 \mathrm{a}$ ). Note that the volume is larger in CG, and the initial position of SNX-482 is $12.5 \mathrm{~nm}$ away from the membrane when compared to the $2.5 \mathrm{~nm}$ for AT MD. Consequently, it takes more than $800 \mathrm{~ns}$ for the toxin to contact the membrane. During this time, SNX-482 is expected to explore all orientations before insertion, thus not being influenced by the initial pose. Nevertheless, we set different initial poses in each of the four CG simulations. In AT, the time to the first toxin-membrane contact was about $300 \mathrm{~ns}$, more likely influenced by the initial pose. However, the gray area depicted in AT simulation trajectory suggests that even at such a short distance, the initial pose was not a relevant factor in the final configuration of the toxin-membrane complex.

To identify residues that contribute to membrane insertion, we computed the average Ip values of the final 180 ns of CG simulation with a 1:1 POPC: POPG membrane (figure

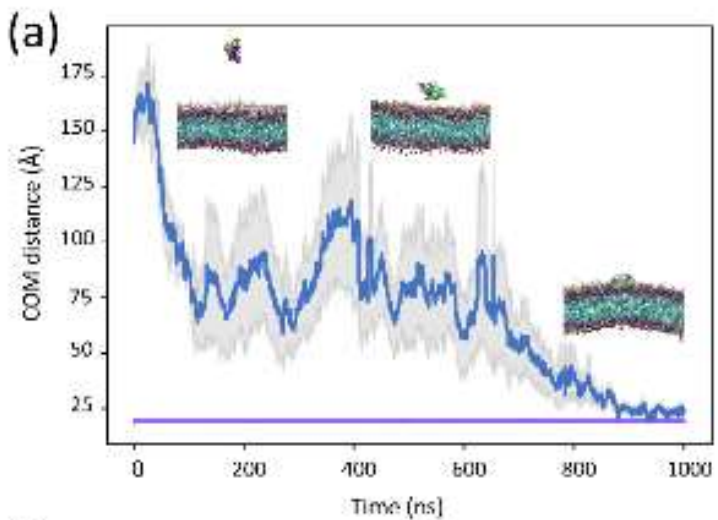

(c)

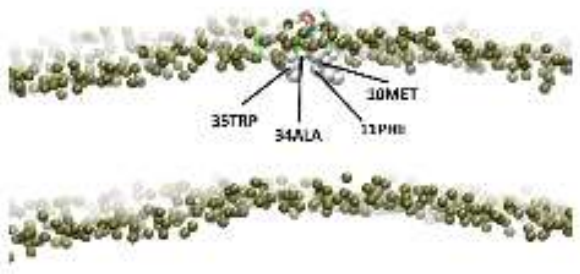

(b)

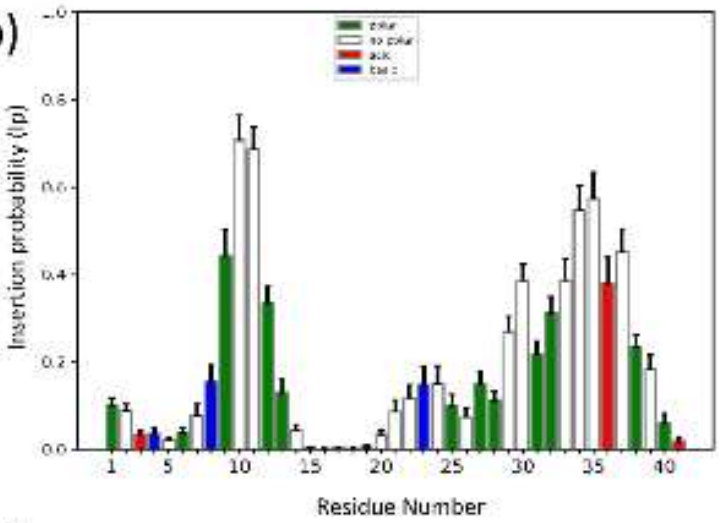

(d)

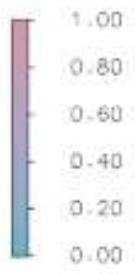

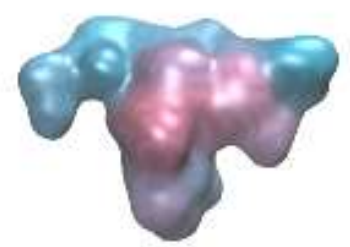

Figure 3. Coarse-grained MD simulations of SNX-482 and POPC: POPG (1:1) (a) Average distance as function of time between the center of Mass (COM) of SNX-482 and the (COM) of the membrane bilayer for four independent simulations. The gray color is the sem error of the distances. The purple line represents the position of the membrane outer leaflet. (b) Average Insertion probabilities (Ip) for each residue. c) Residues that form the hydrophobic face for SNX482 are inserted into the membrane. (d) Surface representation of SNX-482 colored by IP.

3b). We chose the 3:1 POPC:POPG membrane for AT MD for it corresponds to the lipid ratio with the highest IS. In this case, we computed the average Ip for the final $200 \mathrm{~ns}$ (figure 4b). In CG, Met10, Phe11, Phe30, Ala34, Trp35 dwell below the lipids head more than $50 \%$ of the time (figure 3c). This is in close agreement with AT MD, where Tyr9, Met10, Phe11, Leu29, Phe30, Tyr32, Ala34, Trp35 felt below the lipids head more than 60 $\%$ of the time (figure $4 \mathrm{c}$ ). A surface representation of the toxin colored by average Ip 
values (figures $3 \mathrm{~d}$ and $4 \mathrm{~d}$ ) shows that with both CG and all-atom, SNX-482 binding converges to the same pose.

To evaluate the long-term stability of the AT binding pose, we took the last snapshot of one of the POPC: POPG 3:1 simulation and ran it for an additional $2 \mu \mathrm{s}$ in an extended cubic box (see section 2.5). IS values computed in windows of $0.5 \mu$ s exhibit a monotonic increment, reaching a value of 21 in the last window (see supplementary figures S14 and S15), while maintaining the binding pose. Residues 23 to 39 kept increasing their Ip values over time (see supplementary figure S15). A rough linear estimation of the IS timederivative (once bound) yields a value near 2 IS units per microsecond. If residues 8 to 14 and 20 to 40 are the only membrane binders that at equilibrium reach an Ip value near 1, IS maximum would be 26 (see eq. 1). With an IS time-derivative of 2 , an additional $3 \mu \mathrm{s}$ would be necessary to reach a final IS values and, thus, a stable binding pose. Such a slow stabilization of the membrane-toxin complex raises a cautionary note on information derived from short simulations.

The most relevant conclusion of this analysis is that both CG and AT identify a conserved binding mode towards the membrane surface with a preference over partially anionic composition.

3.2. The role of electrostatic interactions in SNX 482 Membrane partitioning.

The total non-bonded energetics of the partitioning process was explored by carrying out two extra $100 \mathrm{~ns}$ simulations of the bound (after $2 \mu \mathrm{s}$ MD simulation) and unbound

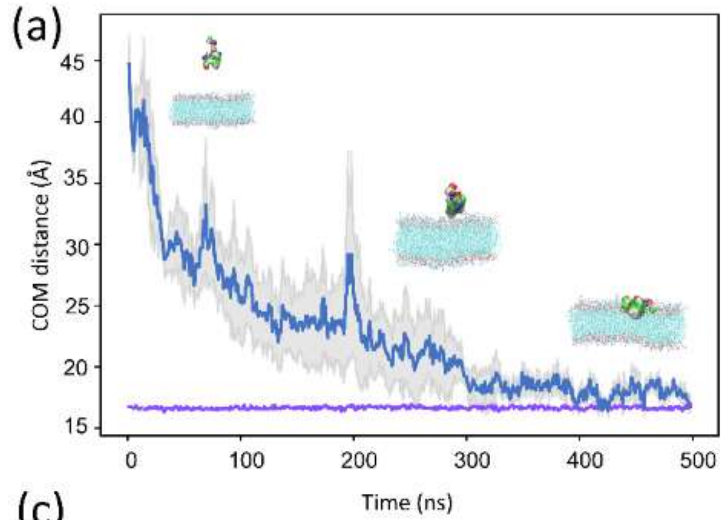

(c)

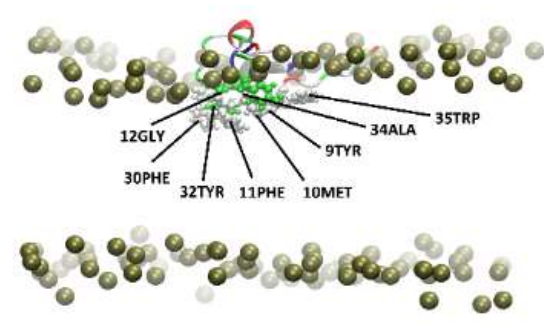

(b)

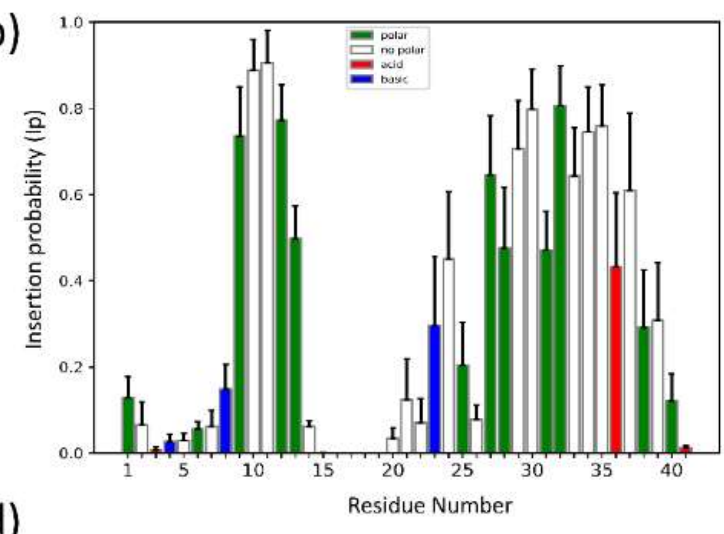

(d)

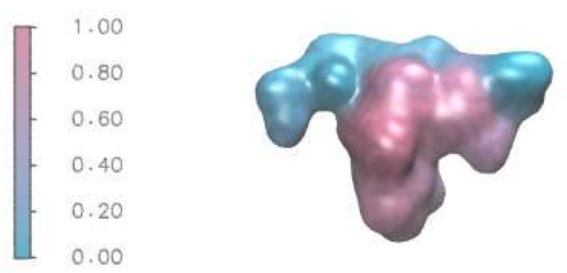

Figure 4. All-atom MD simulations of SNX-482 and POPC: POPG (3:1) (a) Average distance as function of time between the center of Mass (COM) of SNX-482 and the (COM) of the membrane bilayer for four independent simulations. The gray color is the S.E.M. error of the distances. The purple line represents the position of the membrane outer leaflet. (b) Average Insertion probabilities (Ip) for each residue. c) Residues that form the hydrophobic face for SNX482 are inserted into the membrane. (d) Surface representation of SNX-482 colored by IP. 
states for the full-atom POPC:POPG 3:1 system. The toxin was placed at $3 \mathrm{~nm}$ from the outer membrane leaflet to minimize any long-range interaction with the membrane for the unbound state. In addition, we restricted the movement of the toxin along the z-axis to avoid any approximation towards the membrane. The differences in the total nonbonded $\left(\Delta \mathrm{E}_{\mathrm{NB}}\right)$, electrostatic $\left(\Delta \mathrm{E}_{\mathrm{Ele}}\right)$, and Van der Waals $\left(\Delta \mathrm{E}_{\mathrm{VdW}}\right)$ energies between the bound and unbound states of the whole system are presented in Table 2. Clearly, the partition process is energetically favored by electrostatic interactions with an $\Delta$ Eele near -2000 $\mathrm{kcal} / \mathrm{mol}$. The positive $\Delta \mathrm{E}_{\mathrm{VdW}}$, even though there are inserted hydrophobic residues, results from the presence of several polar and charged residues at the membrane surface that come in close contact with the phospholipids' polar heads. Moreover, the release of water molecules to the aqueous phase should also contribute to this value. From the measured partition coefficient for other toxins, the free energy change of membrane binding is in the order of -7 to $-8 \mathrm{Kcal} / \mathrm{mol}$ [12]. This large discrepancy with our calculation of electrostatic energy difference can be attributed to a relevant entropic loss expected from the loss in translational, rotational, and configurational degrees of freedom of the toxin, which is not compensated by the aforementioned release of interfacial water molecules. To explore the precise nature of the membrane-toxin interaction, for the bound state, we computed the pair interaction energy between the toxin and membrane, also shown in Table 2 in their Vdw (EPvdw) and electrostatic (EPele) components. Both terms are favorable, reflecting the polar, charged, and hydrophobic nature of the protein insertion within the membrane. As a whole, these calculations strongly suggest that the partition process is driven by electrostatic interactions that result from the significant number of polar and charged residues that belong to the inserted protein patch (figure $3 \mathrm{~b}$ and supplementary figures S8 and S15).

\subsection{Toxin diffusivity is reduced upon membrane insertion}

To obtain insights on the diffusional effects of membrane partitioning, we computed the diffusion coefficient of the toxin in the aqueous phase $\left(\mathrm{D}_{\mathrm{AP}}\right)$ and the lateral diffusion coefficients (DM) when bound to the membrane for the AT POPC: POPG 3:1 system ( see section 2.5 for more details). Due to finite-size effects, we also extrapolated DM values to macroscopic scales, $\mathrm{D}^{\infty}[32,35]$. These results are presented in Tables 3, S1 and S2 . Bulk diffusion for SNX-482 is one or more than one order of magnitude larger than lateral diffusion within the membrane for the microscopic $\left(\mathrm{D}^{\mathrm{sim}}\right)$ or macroscopic $\left(\mathrm{D}^{\infty}\right)$ estimations, respectively. The latter is expected due to the toxin-membrane interactions and the higher viscosity of the membranous phase. Indeed, a comparison between $\mathrm{D}_{\mathrm{M}}$ values of SNX-482 and the lipids not regarding their composition, reveals that the toxin diffusivity is mostly determined by the membrane, in particular for $\mathrm{D}^{\infty}$ estimations (second column of Table 3). In the context of the two existing binding mechanisms to the channel, this is directly from the bulk or the so-called RD mechanism that involves lateral diffusion along the membrane, it is tempting to carry out a comparison via the estimation of kinetic constants, assuming a diffusion limited phenomenon. However, the latter is not trivial and subject of intense debate in the literature; as for 2D (and 1D as well) processes, these "constants" contain dependencies on time and concentration [10,36,37]. Qualitatively though, the strong reduction in diffusivity within the membrane imposes a kinetic restriction for the RD mechanism as the reduced space needed to encounter the binding partner and effective increase in concentration is compensated by the lower toxin 
mobility, thus suggesting that the key factor in determining which binding mechanism is preferred is the partition coefficient of the toxin.

\section{Conclusions}

In this study and up to our knowledge, for the very first time, the membrane partitioning of the gating-modifier toxin SNX-482 was systematically studied via coarsegrained and all-atom molecular dynamics simulations. We determined that the toxin spontaneously binds to membranes in a conserved manner with a preference over partially anionic membrane composition. The inserted protein patch contains a mixture of polar and hydrophobic residues. Energetic calculations strongly suggest that the partitioning process is driven by electrostatic interactions and that the protein-membrane interactions is stabilized by favorable Van der Waals and electrostatic energies. Lateral diffusion estimations revealed that the toxin mobility within the membrane is at least an order or magnitude slower when compared to bulk conditions which suggest that the key

Table 3. Diffusion coefficients for unbound and bound states

\begin{tabular}{lcc}
\hline \multicolumn{1}{c}{ Diffusion Coefficient } & $\mathrm{D}^{\infty}\left(\mathrm{cm}^{2} / \mathrm{s}\right)$ & $\mathrm{D}^{\mathrm{sim}}\left(\mathrm{cm}^{2} / \mathrm{s}\right)$ \\
\hline $\begin{array}{l}\text { SNX-482 partitioned in POPC:POPG } \\
(3: 1)(\mathrm{DM})^{1}\end{array}$ & $1.1 \times 10^{-7}$ & $4.1 \times 10^{-8}$ \\
SNX-482 in solution (DAP) & - & $1.0 \times 10^{-6}$ \\
POPC
\end{tabular}

$\mathrm{D}^{\infty}$ is the diffusion coefficient extrapolated to and infinite size system. $\mathrm{D}^{\text {sim }}$ is the iffusion coefficient obtained from the AT MD simulations. ${ }^{1}$ Diffusion coefficient in the membrane (DM) was calculated from a $2 \mu$ sT MD in a $16 \mathrm{~nm}$ cubic box. ${ }^{2}$ Diffusion coefficients calculated form 500 ns AT MD simulations

factor which determines the toxin binding mechanism is the partition of the toxin within the membrane.

Supplementary Materials: The following are available online at www.mdpi.com/xxx/s1, Figure S1: title, Table S1: title, Video S1: title.

Author Contributions: G.M, J.A.G and AN designed the research. G.M performed simulations. G.M, J.A.G and A.N wrote the paper. All authors have read and agreed to the published version of the manuscript.

Funding: This work was funded by the National Agency for Research and Development (ANID) through the following programs. Fondecyt grant № 1180987 to J.AG. The CINV and Millennium Nucleus in Nano Biophysics are funded by the Millennium Science Initiative Program ICM-ANID thought grants ICN09-022 (A.N and J.AG) and NCN2021_021 (J.A.G). G.M. participation was funded by ANID BECAS/DOCTORADO NACIONAL 21190530

Data Availability Statement: The data presented in this study are available upon request to the corresponding authors.

Conflicts of Interest: The authors declare no conflict of interest. 


\section{References}

1. Nicholson, G.M. Chapter 63 - Spider Peptides. In Handbook of Biologically Active Peptides (Second Edition); Kastin, A.J., Ed.; Academic Press: Boston, 2013; pp. 461-472 ISBN 978-0-12-385095-9.

2. Avrutina, O. Synthetic Cystine-Knot Miniproteins - Valuable Scaffolds for Polypeptide Engineering. In Protein Targeting Compounds; Böldicke, T., Ed.; Springer International Publishing: Cham, 2016; Vol. 917, pp. 121-144 ISBN 978-3-319-32804-1.

3. Daly, N.L.; Craik, D.J. Bioactive Cystine Knot Proteins. Curr. Opin. Chem. Biol. 2011, 15, 362-368, doi:10.1016/j.cbpa.2011.02.008.

4. Herzig, V.; King, G. The Cystine Knot Is Responsible for the Exceptional Stability of the Insecticidal Spider Toxin $\omega$-HexatoxinHv1a. Toxins 2015, 7, 4366-4380, doi:10.3390/toxins7104366.

5. Agwa, A.J.; Peigneur, S.; Chow, C.Y.; Lawrence, N.; Craik, D.J.; Tytgat, J.; King, G.F.; Henriques, S.T.; Schroeder, C.I. Gating Modifier Toxins Isolated from Spider Venom: Modulation of Voltage-Gated Sodium Channels and the Role of Lipid Membranes. J. Biol. Chem. 2018, 293, 9041-9052, doi:10.1074/jbc.RA118.002553.

6. Catterall, W.A.; Cestèle, S.; Yarov-Yarovoy, V.; Yu, F.H.; Konoki, K.; Scheuer, T. Voltage-Gated Ion Channels and Gating Modifier Toxins. Toxicon 2007, 49, 124-141, doi:10.1016/j.toxicon.2006.09.022.

7. Miller, C.; Moczydlowski, E.; Latorre, R.; Phillips, M. Charybdotoxin, a Protein Inhibitor of Single Ca2+-Activated K+ Channels from Mammalian Skeletal Muscle. Nature 1985, 313, 316-318, doi:10.1038/313316a0.

8. Jung, H.J.; Lee, J.Y.; Kim, S.H.; Eu, Y.-J.; Shin, S.Y.; Milescu, M.; Swartz, K.J.; Kim, J.I. Solution Structure and Lipid Membrane Partitioning of VSTx1, an Inhibitor of the KvAP Potassium Channel + ${ }^{+}$. Biochemistry 2005, 44, 6015-6023, doi:10.1021/bi0477034.

9. Lee, H.C.; Wang, J.M.; Swartz, K.J. Interaction between Extracellular Hanatoxin and the Resting Conformation of the VoltageSensor Paddle in Kv Channels. Neuron 2003, 40, 527-536, doi:10.1016/S0896-6273(03)00636-6.

10. Axelrod, D.; Wang, M.D. Reduction-of-Dimensionality Kinetics at Reaction-Limited Cell Surface Receptors. Biophys. J. 1994, 66, 588-600.

11. Bosmans, F.; Swartz, K.J. Targeting Voltage Sensors in Sodium Channels with Spider Toxins. Trends Pharmacol. Sci. 2010, 31, 175-182, doi:10.1016/j.tips.2009.12.007.

12. Lee, S.-Y.; MacKinnon, R. A Membrane-Access Mechanism of Ion Channel Inhibition by Voltage Sensor Toxins from Spider Venom. Nature 2004, 430, 232-235, doi:10.1038/nature02632.

13. Lau, C.H.Y.; King, G.F.; Mobli, M. Molecular Basis of the Interaction between Gating Modifier Spider Toxins and the Voltage Sensor of Voltage-Gated Ion Channels. Sci. Rep. 2016, 6, 34333, doi:10.1038/srep34333.

14. Deplazes, E.; Henriques, S.T.; Smith, J.J.; King, G.F.; Craik, D.J.; Mark, A.E.; Schroeder, C.I. Membrane-Binding Properties of Gating Modifier and Pore-Blocking Toxins: Membrane Interaction Is Not a Prerequisite for Modification of Channel Gating. Biochim. Biophys. Acta BBA - Biomembr. 2016, 1858, 872-882, doi:10.1016/j.bbamem.2016.02.002.

15. Posokhov, Y.O.; Gottlieb, P.A.; Morales, M.J.; Sachs, F.; Ladokhin, A.S. Is Lipid Bilayer Binding a Common Property of Inhibitor Cysteine Knot Ion-Channel Blockers? Biophys. J. 2007, 93, L20-L22, doi:10.1529/biophysj.107.112375.

16. Newcomb, R.; Chen, X.; Dean, R.; Dayanithi, G.; Cong, R.; Szoke, B.; Lemos, J.; Bowersox, S.; Miljanich, G. SNX-482: A Novel Class E Calcium Channel Antagonist from Tarantula Venom. CNS Drug Rev. 2006, 6, 153-173, doi:10.1111/j.15273458.2000.tb00143.x.

17. Newcomb, R.; Szoke, B.; Palma, A.; Wang, G.; Chen, X.; Hopkins, W.; Cong, R.; Miller, J.; Urge, L.; Tarczy-Hornoch, K.; et al. Selective Peptide Antagonist of the Class E Calcium Channel from the Venom of the Tarantula Hysterocrates Gigas ${ }^{\dagger}$. Biochemistry 1998, 37, 15353-15362, doi:10.1021/bi981255g.

18. Bourinet, E.; Stotz, S.C.; Spaetgens, R.L.; Dayanithi, G.; Lemos, J.; Nargeot, J.; Zamponi, G.W. Interaction of SNX482 with Domains III and IV Inhibits Activation Gating of A1E (CaV2.3) Calcium Channels. Biophys. J. 2001, 81, 79-88, doi:10.1016/S00063495(01)75681-0.

19. Kimm, T.; Bean, B.P. Inhibition of A-Type Potassium Current by the Peptide Toxin SNX-482. J. Neurosci. 2014, 34, 9182-9189, doi:10.1523/JNEUROSCI.0339-14.2014.

20. Munhoz, J.; Thomé, R.; Rostami, A.; Ishikawa, L.L.W.; Verinaud, L.; Rapôso, C. The SNX-482 Peptide from Hysterocrates Gigas Spider Acts as an Immunomodulatory Molecule Activating Macrophages. Peptides 2021, 146, 170648, doi:10.1016/j.peptides.2021.170648.

21. Johnson, M.; Zaretskaya, I.; Raytselis, Y.; Merezhuk, Y.; McGinnis, S.; Madden, T.L. NCBI BLAST: A Better Web Interface. Nucleic Acids Res. 2008, 36, W5-9, doi:10.1093/nar/gkn201.

22. Eswar, N.; Webb, B.; Marti-Renom, M.A.; Madhusudhan, M.S.; Eramian, D.; Shen, M.; Pieper, U.; Sali, A. Comparative Protein Structure Modeling Using Modeller. Curr. Protoc. Bioinforma. 2006, 15, 5.6.1-5.6.30, doi:10.1002/0471250953.bi0506s15.

23. Scott, W.R.P.; Hünenberger, P.H.; Tironi, I.G.; Mark, A.E.; Billeter, S.R.; Fennen, J.; Torda, A.E.; Huber, T.; Krüger, P.; van Gunsteren, W.F. The GROMOS Biomolecular Simulation Program Package. J. Phys. Chem. A 1999, 103, 3596-3607, doi:10.1021/jp984217f.

24. Van Der Spoel, D.; Lindahl, E.; Hess, B.; Groenhof, G.; Mark, A.E.; Berendsen, H.J.C. GROMACS: Fast, Flexible, and Free. J. Comput. Chem. 2005, 26, 1701-1718, doi:10.1002/jcc.20291. 
25. Souza, P.C.T.; Alessandri, R.; Barnoud, J.; Thallmair, S.; Faustino, I.; Grünewald, F.; Patmanidis, I.; Abdizadeh, H.; Bruininks, B.M.H.; Wassenaar, T.A.; et al. Martini 3: A General Purpose Force Field for Coarse-Grained Molecular Dynamics. Nat. Methods 2021, 18, 382-388, doi:10.1038/s41592-021-01098-3.

26. Parrinello, M.; Rahman, A. Polymorphic Transitions in Single Crystals: A New Molecular Dynamics Method. J. Appl. Phys. 1981, 52, 7182-7190, doi:10.1063/1.328693.

27. de Jong, D.H.; Singh, G.; Bennett, W.F.D.; Arnarez, C.; Wassenaar, T.A.; Schäfer, L.V.; Periole, X.; Tieleman, D.P.; Marrink, S.J. Improved Parameters for the Martini Coarse-Grained Protein Force Field. J. Chem. Theory Comput. 2013, 9, 687-697, doi:10.1021/ct300646g.

28. Lee, J.; Cheng, X.; Swails, J.M.; Yeom, M.S.; Eastman, P.K.; Lemkul, J.A.; Wei, S.; Buckner, J.; Jeong, J.C.; Qi, Y.; et al. CHARMMGUI Input Generator for NAMD, GROMACS, AMBER, OpenMM, and CHARMM/OpenMM Simulations Using the CHARMM36 Additive Force Field. J. Chem. Theory Comput. 2016, 12, 405-413, doi:10.1021/acs.jctc.5b00935.

29. Phillips, J.C.; Braun, R.; Wang, W.; Gumbart, J.; Tajkhorshid, E.; Villa, E.; Chipot, C.; Skeel, R.D.; Kalé, L.; Schulten, K. Scalable Molecular Dynamics with NAMD. J. Comput. Chem. 2005, 26, 1781-1802, doi:10.1002/jcc.20289.

30. Huang, J.; MacKerell, A.D. CHARMM36 All-Atom Additive Protein Force Field: Validation Based on Comparison to NMR Data. J. Comput. Chem. 2013, 34, 2135-2145, doi:10.1002/jcc.23354.

31. Ryckaert, J.-P.; Ciccotti, G.; Berendsen, H.J.C. Numerical Integration of the Cartesian Equations of Motion of a System with Constraints: Molecular Dynamics of n-Alkanes. J. Comput. Phys. 1977, 23, 327-341, doi:10.1016/0021-9991(77)90098-5.

32. Venable, R.M.; Ingólfsson, H.I.; Lerner, M.G.; Perrin, B.S.; Camley, B.A.; Marrink, S.J.; Brown, F.L.H.; Pastor, R.W. Lipid and Peptide Diffusion in Bilayers: The Saffman-Delbrück Model and Periodic Boundary Conditions. J. Phys. Chem. B 2017, 121, 34433457, doi:10.1021/acs.jpcb.6b09111.

33. Agwa, A.J.; Peigneur, S.; Chow, C.Y.; Lawrence, N.; Craik, D.J.; Tytgat, J.; King, G.F.; Henriques, S.T.; Schroeder, C.I. Gating Modifier Toxins Isolated from Spider Venom: Modulation of Voltage-Gated Sodium Channels and the Role of Lipid Membranes. J. Biol. Chem. 2018, 293, 9041-9052, doi:10.1074/jbc.RA118.002553.

34. Swartz, K.J.; MacKinnon, R. An Inhibitor of the Kv2.1 Potassium Channel Isolated from the Venom of a Chilean Tarantula. Neuron 1995, 15, 941-949, doi:10.1016/0896-6273(95)90184-1.

35. Camley, B.A.; Lerner, M.G.; Pastor, R.W.; Brown, F.L.H. Strong Influence of Periodic Boundary Conditions on Lateral Diffusion in Lipid Bilayer Membranes. J. Chem. Phys. 2015, 143, 243113, doi:10.1063/1.4932980.

36. Hardt, S.L. Rates of Diffusion Controlled Reactions in One, Two and Three Dimensions. Biophys. Chem. 1979, 10, 239-243, doi:10.1016/0301-4622(79)85012-7.

37. Goldstein, B.; Dembo, M. Approximating the Effects of Diffusion on Reversible Reactions at the Cell Surface: Ligand-Receptor Kinetics. Biophys. J. 1995, 68, 1222-1230, doi:10.1016/S0006-3495(95)80298-5. 